\title{
Diafanización Dental de Cuatro Especies de Seláceos (Carcharhinus leucas, Galeocerdo cuvier, Rhizoprionodon longurio y Sphyrna sp)
}

\author{
Dental Diaphonization of Four Salaceos Species (Carcharhinus leucas, \\ Galeocerdo Cuvier, Rhizoprionodon longurio and Sphyrna sp)
}

\author{
Génesis Ariadna Gutiérrez Pech ${ }^{1}$ \& GabrielaSánchez Fabila ${ }^{1}$; Roberto Moreno Colín ${ }^{1}$; Luis Fernando \\ Del Moral Flores²; Iris de los Ángeles Rodríguez Trinidad ${ }^{3} \&$ Francisco Torres Salazar $^{3}$
}

GUTIÉRREZ, P. G. A.; SÁNCHEZ, F. G.; MORENO, C. R.; DEL MORAL, F. L. F.; RODRÍGUEZ, T. I. A. \& TORRES, S. F. Diafanización dental de cuatro especies de seláceos (Carcharhinus leucas, Galeocerdo cuvier, Rhizoprionodon longurio y Sphyrna sp). Int. J. Morphol., 38(4):970-974, 2020.

RESUMEN: El estudio morfológico de la dentadura de chondrichthyes representa un carácter taxonómico importante empleado para la clasificación e identificación de diferentes especies. Se diafanizaron dientes de cuatro especies distintas de selacimorfos (Carcharhinus leucas, Galeocerdo cuvier, Rhizoprionodon longurio y Sphyrna sp.) con la finalidad de estandarizar una técnica dental para su transparentación. Estandarizando la técnica de Okumura-Aprile aplicada para la diafanización dental de humanos, se obtuvo una diafanización óptima en las cuatro especies en tratamiento con $\mathrm{HCl}$ al 7 \% donde se podía observar con claridad la cámara pulpar, por lo que podemos concluir que la técnica de Okumura-Aprile es eficiente en la diafanización dental de tiburones.

PALABRAS CLAVE: Diafanización; Vertebrados; Seláceos; Dientes; Morfometría dental.

\section{INTRODUCCIÓN}

Las mandíbulas y la dentición de los lamniformes representan un carácter taxonómico importante empleado para la clasificación e identificación de diferentes especies (Sadowsky, 1969). Los dientes en elasmobranquios están unidos a la mandíbula por capas de tejido conectivo y pueden ser reemplazados en cualquier etapa del desarrollo, tienen diversas formas, que oscilan entre puntas curvadas y puntas triangulares planas, esto dependiendo de su alimentación y relación evolutiva (Gajic, 2013). El tipo de dientes es muy importante porque los tiburones no mastican su alimento solo lo sujetan, lo desgarran y lo tragan, como no tienen raíces que los unan a las mandíbulas algunos se van cayendo.

Así, los caracteres dentales constituyen una gran ayuda en la diagnosis taxonómica haciéndola más detallada y acabada (Bass, 1975). En el caso del tiburón toro Carcharhinus leucas (Müller \& Henle 1839), por ejemplo, los dientes superiores son triangulares y aserrados con cúspides anchas, mientras que los dientes de la mandíbula inferior son robustos de cúspides verticales a ligeramente oblicuas con márgenes aserrados; mientras que en el tiburón tigre Galeocerdo cuvier (Péron \& Lesueur 1822) los dientes superiores e inferiores son muy similares, aserrados con márgenes externos escotados y las puntas oblicuamente hacia afuera; el tiburón limón (Negaprion brevirostris Poey 1868) con dientes triangulares, delgados, y aserrados en la base de la corona; y por último, el cazón Rhizoprionodon longurio (Jordan \& Gilbert 1882), con los dientes de ambas mandíbulas con cúspides oblicuas (Castellanos et al., 2013; De Moral Flores et al., 2013).

Las técnicas de diafanización dental, son muy variables y han sido frecuentemente empleadas para evaluar diferentes aspectos anatómicos y endodónticos, como la dureza similar al diente en estado natural. La diafanización dental consta de cuatro pasos: preparación de las muestras (tinción de los conductos radiculares), desmineralización, deshidratación y transparentación (Greco-Machado et al., 2008).

En 1917 Okumura llevó esta técnica a dientes humanos y realizó un estudio exhaustivo sobre la anatomía interna de estos gracias a la diafanización, siendo el primero en clasificar los canales radiculares de acuerdo a su distribu-

\footnotetext{
${ }^{1}$ Laboratorio de Anatomía de Vertebrados y Educación Científica, Facultad de Estudios Superiores Iztacala UNAM, México.

${ }^{2}$ Laboratorio de Zoología, Facultad de Estudios Superiores Iztacala UNAM, México

${ }^{3}$ Laboratorio de Estomatológicas y Patología Oral, Facultad de Estudios Superiores Iztacala UNAM, México.
} 
ción anatómica. Para lograr esto, agregó a su técnica la inyección de tinta china en el interior de la cavidad pulpar y así lograr hacer evidente el sistema de canales radiculares (Bravo et al., 2015). Esta técnica ha sido empleada principalmente en dientes de organismos óseos, pero en el caso de organismos cartilaginosos, como los tiburones, no hay estudios que avalen la efectividad de un método en particular.

Debido a la poca información reportada acerca de la anatomía dental y a la importancia económica, biológica, trófica, cultural y médica que representan los tiburones en México, el realizar un estudio descriptivo de las estructuras taxonómicas principales tales como la dentición, a través de la diafanización permiten proporcionar información que contribuya a ampliar el conocimiento anatómico que hay en la actualidad y sea útil para futuros estudios. Por lo que los objetivos del presente trabajo son; Describir la forma de los conductos radiculares de dientes de cuatro distintas especies de tiburones a través de la técnica de diafanización dental, estandarización de la diafanización dental para tiburones basada en la técnica de Okumura-Aprile.

\section{MATERIAL Y MÉTODO}

Se utilizaron 96 dientes distribuidos de la siguiente manera 28 dientes de tiburón cazón, 28 dientes de T. toro, 28 dientes de tiburón tigre y 12 de tiburón martillo. Todos ellos fueron extraídos de las mandíbulas, posteriormente se dividieron en dos grupos, el primero para sumergirlo en ácido clorhídrico $\mathrm{HCl}$ al $6 \%$ y el segundo al $7 \%$ permaneciendo en tratamiento de 3, 6, 9, 12, 1518 y 24 horas, con dos repeticiones en cada periodo, con la finalidad de desmineralizar estos. Posteriormente se realizó un lavado de los dientes en agua corriente durante 10 horas. Se sometieron los dientes a un tren de etanol para deshidratarlos desde el 70, 80, 90 y $96 \%$. Antes de empezar el proceso de diafanización se inyectó tinta china de marca pelikan en la cámara pulpar de los dientes durante dos horas dentro de una cámara de vacío, para después lavarlos con agua corriente. Para la diafanización se decidió aumentar el periodo de tiempo a 32 y 48 horas bajo el tratamiento de $7 \%$ de $\mathrm{HCl}$, con una repetición para cada periodo, continuando con la técnica previamente descrita.

\section{RESULTADOS}

A continuación, se presentan los resultados de la diafanización de dientes, primero en la concentración del $6 \%$ (Fig. 1) y después en la de $7 \%$ (Fig. 2).
Las regiones de los dientes que se diafanizaron dieron la zona apical, las cúspides primarias y laterales en el caso del tiburón martillo, la región apical y las cuspides laterales para el tiburon toro, la medial y distal en el caso del tiburón limón y el márgen lingual y labial en el caso del cazón.

La región que se conserva de un color crema o amarillo muy claro son las zanas basales principalmente el pie de la corona, el foramen central, y la región mesial. En las siguientes imágenes se observan los dientes de las cuatro especies, cazón en la parte superior izquierda, martillo en la parte superior derecha, toro en la porción inferior izquierda y tigre en la inferior derecha. Para la concentración del $6 \%$ a las 3 horas; cómo se puede observar los dientes se van desmineralizando, empiezan a verse translucidos sobre todo en la parte más externa del diente; mientras que el pie de la corona se encuentra en un color crema y la parte central del diente sigue blanquecina. A las nueve horas se presenta un mayor contraste en la diafanización, sobre todo en dientes de tiburón se comienzan a observar los espacios donde se desarrollarán los conductos radiculares en organismos más complejos.

A las 15 horas los dientes del tiburón tigre ya se encuentran completamente diafanizados y con la cámara pulpar amarillo claro, también se puede observar en los dientes. Que hay un triángulo ligeramente marcado y blanquecino hacia la parte apical.

Esto es importante por que como se menciona en la literatura en el caso de tiburones que tienen dientes muy angostos hacia la zona apical son tiburones que difícilmente atacan a las personas porque sus dientes están diseñados para sujetar a la presa, mientras que en los dientes de tiburones como el tigre son dientes que están desarrollados de una forma más robusta, aserrados pues son para desgarran a la presa. Al diafanizar los dientes podemos confirmar esto por el tipo de cámara pulpar es más grande y llega hasta la zona apical del diente (Mendoza-Vargas, 2008).

A las 24 horas del tratamiento podemos observar que los dientes estan bien translucidos.

Al $7 \%$ lo que podemos observar es que a las tres horas los dientes presentan un halo alrededor como si la diafanización fuera de exterior a interior. En esta foto pareciera que el $6 \%$ tuviese mejor efecto al inicio que al 7 $\%$; sin embargo, si observamos los dientes a las nueve horas, podemos mencionar que no hay cambio que se encuentran casi iguales a los de las tres horas, en las siguientes muestras podemos observar cambios más significativos. 


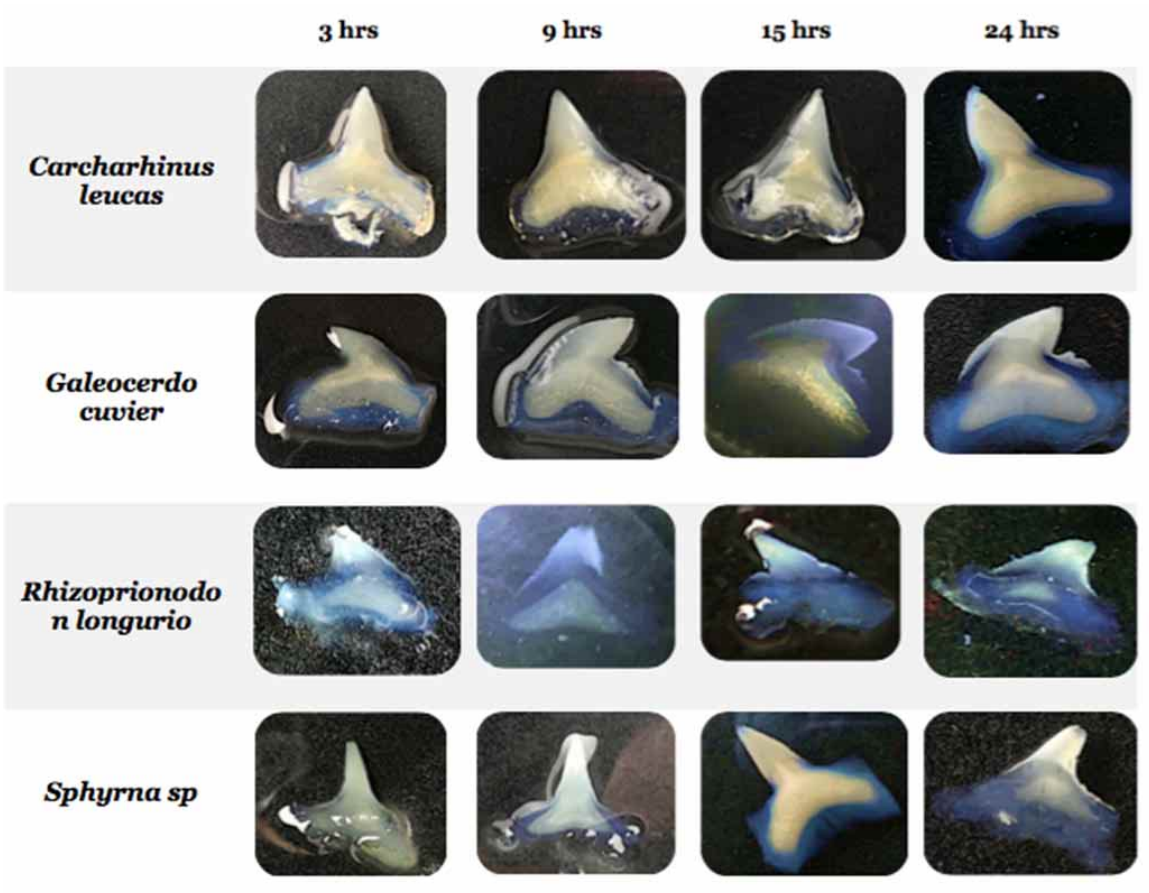

Fig. 1. Grupo de dientes correspondientes al proceso de diafanización con ácido clorhídrico $(\mathrm{HCl})$ al $6 \%$.

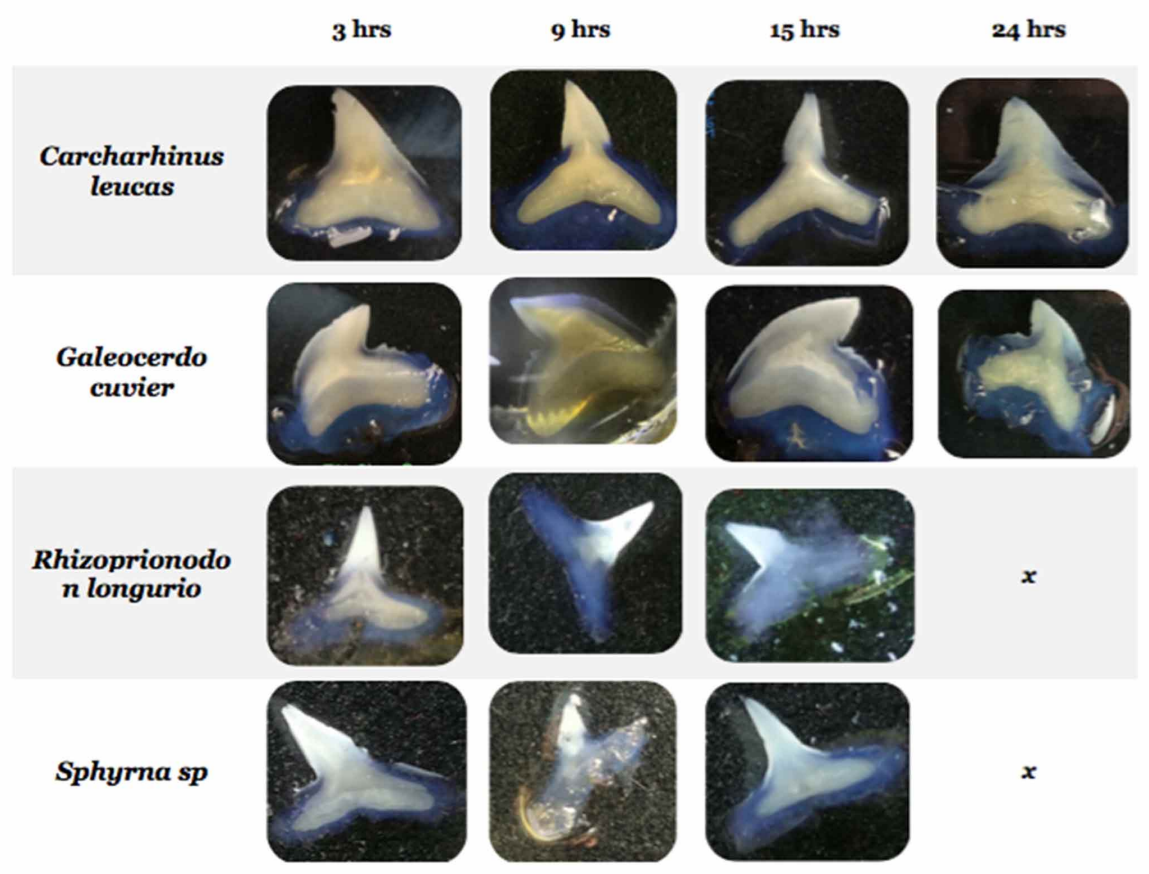

Fig. 2. Grupo de dientes correspondientes al proceso de diafanización con ácido clorhídrico $(\mathrm{HCl})$ al $7 \%$.
A las 15 horas si vemos la imagen ya se encuentran más translucidos (Fig. 2).

Al realizar la prueba estadística no paramétrica de KruskalWallis para los datos del primer tratamiento con $\mathrm{HCl}$ al $6 \%$ con un valor $\mathrm{Xi}^{2,,}=8,597$ y $\mathrm{p}=0,2327$ se determina que hay una diferencia significativa entre los valores de las medias para la diafanización para cada una de las especies; mientras que en el tratamiento con $\mathrm{HCl}$ al $7 \%$ con valores de $\mathrm{Xi}^{2}=0,5294$ y $\mathrm{p}=0,8978$ no hay una diferencia significativa entre los valores de las medias.

En los dientes de C. leucas y G. cuvier que fueron teñidos con tinta china se identifica claramente las estructuras internas (Fig. 3). En la fotografía de la pieza dental de $G$. cuvier se observa una cámara pulpar extendida desde la parte basal en la raíz hasta la zona más apical de la cúspide primaria. Entre la raíz y la cámara pulpar se observan los canales radiculares, que son las estructuras nerviosas del diente. Mientras que en $C$. leucas la cámara pulpar es de menor tamaño, aunque los canales radiculares se encuentran en mayor cantidad, por lo que se asume que $C$. leucas es mucho más sensible a la sensación de la pérdida de piezas por la polifiodontía, que $G$. cuvier.

Se realizó un estudio histopatológico que permitió identificar estructuras dentales morfodiferenciadas e histodiferenciadas, en donde se observa la presencia de vestigios de una estructura externa conformada por esmalte, seguida de dentina palial y circumpulpar, pudiéndose observar la presencia de túbulos dentinarios. Al interior se identifica la presencia de una cavidad conteniendo la pula cameral con zonas de osteodentina. 

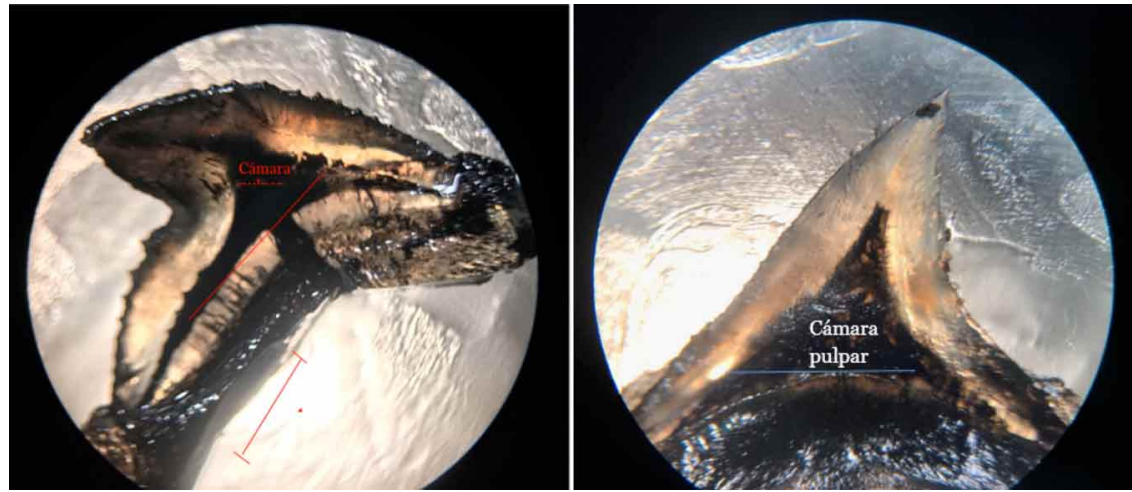

Fig. 3. A y B. Dientes de Galeocerdo cuvier y Carcharhinus leucas diafanizados y teñidos con tinta china observados a 40x en un microscopio estereoscópico, en la imagen de la izquierda se pueden observar inervaciones nerviosas que probablemente generen una sensibilidad en los dientes al perderlos.
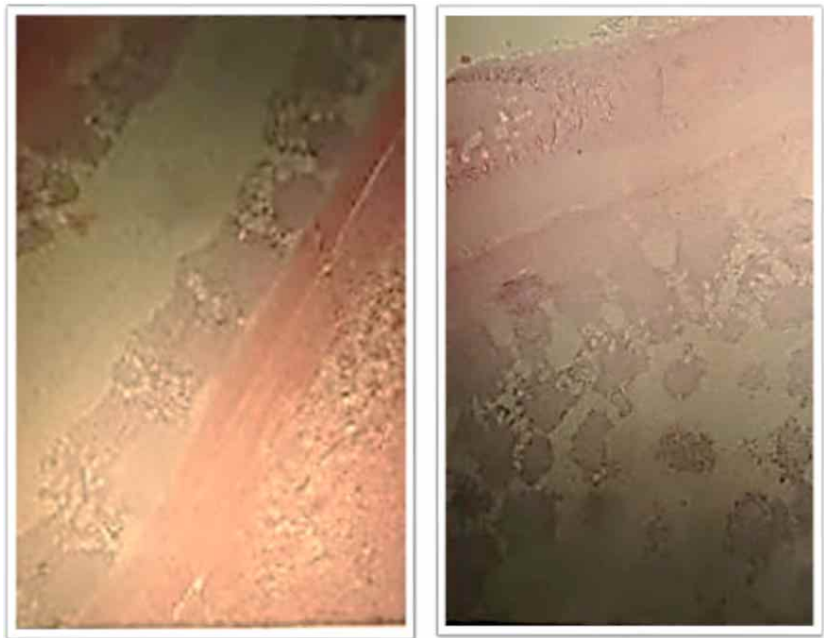

Fig. 4. A y B. Tejidos estructurales de las piezas dentales de seláceos.

\section{DISCUSIÓN}

La composición de fluorapatita de los dientes de tiburón, presente en altas concentraciones en el enameloide (capa más externa de recubrimiento), y en menor cantidad en la dentina, a comparación de los dientes de mamíferos, otorga una mayor dureza y resistencia a agentes patógenos y químicos ácidos (Enax et al., 2012). En los resultados obtenidos se observa que las cúspides en la parte apical no se transparentan completamente a diferencia de dientes de humanos con contenido de hidroxiapatita. La hidroxiapatita es soluble en ácidos debido a que tanto el PO43- como el OHreaccionan con $\mathrm{H}+$.

La fluorapatita es otro mineral fosfato, soluble en ácido clorhídrico, no obstante; necesita una mayor concen- tración y tiempo de exposición al ácido para lograr diafanizarse. Por lo tanto, para futuras investigaciones se recomienda ampliar el tiempo de la descalcificación y aplicar como aclarante otra sustancia química diferente al salicilato de metilo, como el xilol, que permita conservar lo más posible la dureza del diente, ya que el tiempo de exposición prolongado en el ácido puede provocar que partes de la morfología del diente se pierdan (Blancas, 2001).

Se puede observar que la diafanización se ve con mayor claridad en los dientes de mayor tamaño pertenecientes a Galeocerdo cuvier y Carcharias taurus, esto puede ser por la diferencia en la etapa de desarrollo de los tiburones, ya que durante esta investigación no se determinó si las muestras dentales eran pertenecientes a seláceos juveniles o adultos en las especies de Sphyrna sp. y Rhizoprionodon longurio.

En el tratamiento con $\mathrm{HCl}$ al $7 \%$ en las especies Sphyrna sp. y Rhizoprionodon longurio, la calidad de la diafanización disminuye una vez que incrementa el tiempo de exposición. Esto puede ser debido a que los dientes llevaban un periodo largo de tiempo de ser extraídos, por lo cual el tejido se seca y no reacciona de la misma manera con los químicos utilizados a diferencia de un tejido fresco (Blancas).

Los dientes de los tiburones no están implantados en alvéolos; están simplemente insertados en el tegumento y se mantienen en su sitio por su raíz, que corresponde a la placa basal de los dentículos dérmicos. En la tinción de la cámara pulpar mediante la inyección de tinta china en ejemplares de Galeocerdo cuvier y Carcharias taurus se puede observar la cámara pulpar, así como algunas líneas paralelas situadas entre la raíz, la dentina y el enameloide, haciendonos pensar que los tiburones pueden tener sensibilidad al perder los dientes. El desarrollo de más estudios enfocados a estas estructuras, así como estudios en donde se busque la presencia de canales radiculares permitirá ampliar el conocimiento respecto a la polifiodonta, y sus implicaciones evolutivas.

De acuerdo con las observaciones realizadas en el estudio histopatológico, se determinaron las estructuras principales del tejido de los dientes de seláceos. La estructura más externa del diente se reconoce como el esmalte, segui- 
do por la dentina palial y circumpular. La dentina palial es la primera capa que se forma cerca del esmalte, mientras que la dentina circumpulpar marca el límite entre la cámara pulpar o pulpa cameral. Dentro de la dentina, se encuentran los túbulos dentinarios o conductos radiculares, que son las estructuras que conectan el nervio contenido dentro de la cámara pulpar con el esmalte, permitiendo la sensibilidad a cambios extrínsecos (Brännström, 1984). Por último, la pulpa cameral presenta una última estructura denominada como "osteodentina" o "dentina traumática". Esta se forma debido a que después de la pérdida de una pieza dental la estructura se deposita tan rápidamente que atrapa células o tejidos, dándole una apariencia osteoide (Cabrera, 2008).

\section{CONCLUSIÓN}

El método de diafanización de Okumura-Aprile resultó ser efectivo para la diafanización de dientes de seláceos. Siendo que el tratamiento con ácido clorhídrico al $7 \%$ el más efectivo para Galeocerdo cuvier y Carcharhinus leucas, mientras que el tratamiento con ácido clorhídrico al $6 \%$ en el periodo de 24 hr tuvo una calidad de diafanización óptima en las otras especies de tiburones. Sin embargo, debido a que la transparencia de los dientes difiere según la concentración y el tiempo de exposición en el descalcificador utilizado, se sugiere que se amplíe el tiempo de exposición en el caso de utilizar ácido clorhídrico con concentraciones del $7 \%$ para lograr una transparentación total.

Podemos concluir que gracias a la tinción de los dientes con tinta china se puede observar una serie de ramificaciones nerviosas que pudiesen provocar sensibilidad al perderlos.

La tinción con tinta china de las piezas dentales, así como el estudio histopatológico permitieron observar las estructuras histológicas internas, así como facilitar su identificación y función.

GUTIÉRREZ, P. G. A.; SÁNCHEZ, F. G.; MORENO, C. R.; DEL MORAL, F. L. F.; RODRÍGUEZ, T. I. A. \& TORRES, S. F. Dental diaphonization of four seláceos species (Carcharhinus leucas, Galeocerdo cuvier, Rhizoprionodon longurio and Sphyrna sp). Int. J. Morphol., 38(4):970-974, 2020.

The morphological study of the chondrichthyes teeth represents an important taxonomic characteristic used for the classification and identification of different species. The teeth of four different species of selacimorphs (Carcharhinus leucas, Galeocerdo cuvier, Rhizoprionodon longurio and Sphyrna sp.) were diaphonized in order to standardize a dental technique for their transparency. By standardizing the Okumura-Aprile technique applied for the dental diaphonization of humans, an optimal diaphonization was obtained in the four species treated with $7 \% \mathrm{HCl}$ where the pulp chamber was clearly observed. Therefore, we may conclude that the OkumuraAprile technique is efficient in shark dental diaphonization.

KEY WORDS: Dental diaphonization technique; Okumura-Aprile; Vertebrates; Sharks; Chondrichthyes; Tooth morphometry.

\section{REFERENCIAS BIBLIOGRÁFICAS}

Bass, A. J.; D'aubrey, J. D. \& Kistnasamy, N. Sharks of the east coast of Southern Africa. III. The families Carcharhinidae (excluding Mustelus and Carcharhinus) and Sphyrnidae. Investig. Rep. Oceanogr. Res. Inst., 38:1-100, 1975.

Blancas, F. Transparentación Dental (Diafanización) como Método de Enseñanza Preclínica en el Área de Endodoncia. Tesina de Licenciatura (Cirujano Dentista). Ciudad de México, Facultad de Odontología, Universidad Nacional Autónoma de México, 2001. pp.3-32.

Brännström, M. Communication between the oral cavity and the dental pulp associated with restorative treatment. Oper. Dent., 9(2):57-68, 1984.

Bravo, R.; Valenzuela, M.; Cáceres, F. \& Soto, R. Application of potassium hydroxide and glycerin technique for dental clearing. Int. J. Morphol., 33(2):673-7, 2015

Cabrera, M. Dentina Terciaria o de Reparación Inducida por Extracto de Caléndula Officinalis Linn Europea en Molares de Ratas Norvegicus Albinus. Tesis Doctoral. Sevilla, Universidad de Sevilla, 2008.

Castellanos, J.; Ramírez, C. \& Castillo, J. Catálogo de Aletas, Tronchos y Cabezas de Tiburones del Pacífico Mexicano. Ciudad de México, Instituto Nacional de Pesca, SAGARPA, 2013. pp.18, 24, 40.

De Moral Flores, L. F. \& Pérez Ponce de Léon, G. Tiburones, rayas y quimeras del México. CONABIO. Biodiversitas, 111:1- 6, 2013. Disponible en: https://www.biodiversidad.gob.mx/Biodiversitas/Articulos/ biodiv111art1.pdf

Enax, J.; Prymak, O.; Raabe, D. \& Epple, M. Structure, composition, and mechanical properties of shark teeth. J. Struct. Biol., 178(3):290-9, 2012.

Gajic, A. Comparative Odontology of Selachians (Chondrichthyes: Elasmobranchii): Developmant and Morphological Characteristic of Teeth. Sheffield, SymbioSE 2013, The 17th Anual Symposium Students in Europe, at The University of Sheffield, Department of Molecular Biology, The Book of Abstracts, 31, 2013.

Greco-Machado, Y.; García-Molina, J. A.; Bueno-Martínez, R.; ManzanaresCéspedes, M. C. \& Lozano-De Luaces, V. Técnicas de diafanización: estudio comparativo. Endodoncia, 26(2):85-92, 2008.

Mendoza-Vargas, O. Dentición en Tiburones. Ciudad de México, Tercer Simposium Nacional de Tiburones y Rayas. Ciudad Universitaria, Universidad Nacional Autónoma de México, 2008.

Sadowsky, V. On the dentition of the sand shark, Odontaspis taurus, from the vicinity of Cananéia, Brazil. Bol. Inst. Oceanogr., 18(1):37-44, 1969.

Dirección para correspondencia:

Dra. Gabriela Sánchez Fabila

Laboratorio de anatomía de Vertebrados y

Educación Científica

Facultad de Estudios Superiores Iztacala UNAM

MÉXICO

Email: gsfabila@gmail.com

Recibido : 13-02-2020

Aceptado: $25-03-2020$ 